\title{
The X-ray and radio connection in low-luminosity active nuclei
}

\author{
F. Panessa ${ }^{1}$, X. Barcons ${ }^{1}$, L. Bassani ${ }^{2}$, M. Cappi ${ }^{2}$, F. J. Carrera ${ }^{1}$, L. C. Ho ${ }^{3}$, and S. Pellegrini ${ }^{4}$ \\ 1 Instituto de Física de Cantabria (CSIC-UC), Avda. de los Castros, 39005 Santander, Spain \\ e-mail: panessa@ifca.unican.es \\ 2 INAF - IASF, via P. Gobetti 101, 40129 Bologna, Italy \\ 3 The Observatories of the Carnegie Institution of Washington, 813 Santa Barbara St., Pasadena, CA 91101, USA \\ 4 Dipartimento di Astronomia, Università di Bologna, via Ranzani 1, 40127 Bologna, Italy
}

Received 15 December 2006 / Accepted 16 January 2007

\section{ABSTRACT}

\begin{abstract}
We present the results of the correlation between the nuclear 2-10 keV X-ray and radio (at $2 \mathrm{~cm}, 6 \mathrm{~cm}$, and $20 \mathrm{~cm}$ ) luminosities for a well-defined sample of local Seyfert galaxies. We use a sample of low luminosity radio galaxies (LLRGs) for comparison. In both Seyfert and LLRG samples, X-ray and radio luminosities are significantly correlated over 8 orders of magnitude, indicating that the X-ray and radio emission sources are strongly coupled. Moreover, both samples show a similar regression slope, $L_{\mathrm{X}} \propto L_{\mathrm{R}}^{0.97}$, but Seyfert galaxies are three orders of magnitude less luminous in the radio band than LLRGs. This suggests that either similar physical mechanisms are responsible for the observed emission or a combination of different mechanisms ends up producing a similar correlation slope. Indeed, the common belief for LLRG is that both the X-ray and radio emission are likely dominated by a relativistic jet component, while in Seyfert galaxies the X-ray emission probably arises from a disk-corona system and the radio emission is attributed to a jet/outflow component. We investigate the radio loudness issue in the two samples and find that the Seyfert galaxies and the LLRGs show a different distribution of the radio loudness parameters. No correlation is found between the luminosity and the radio loudness; however, the latter is related to the black hole mass and anti-correlated with the Eddington ratio. The dichotomy in the radio loudness between Seyfert and LLRG observed down to low Eddington ratios, $L_{2-10 \mathrm{keV}} / L_{\mathrm{Edd}} \sim 10^{-8}$, does not support the idea that the origin of the radio loudness is due to a switch in the accretion mode.
\end{abstract}

Key words. accretion, accretion disks - X-rays: galaxies - galaxies: Seyfert - galaxies: nuclei

\section{Introduction}

Recent developments have shown that all active galactic nuclei (AGN) are radio sources at some level. Indeed, a high fraction of radio cores have been detected in radio-quiet AGN and their radio emission probably results from some sort of outflow of radio plasma from the nucleus, in the form of jets, bubbles or plasmoids (Wilson \& Ulvestad 1987; Pedlar et al. 1985). The issue that all AGN could host a jet and be synchrotron emitters is in apparent contrast with the sharp division observed between radio-loud and radio-quiet AGN. Radio-loud sources constitute only $\sim 10-20 \%$ of the AGN population (Strittmatter et al. 1980; Kellermann et al. 1989). Optically selected AGN are historically divided as being radio-loud or radio-quiet depending on the value of the radio loudness parameter $R$, defined as the ratio between the monochromatic luminosities at radio and optical frequencies $\left(R \equiv L_{6 \mathrm{~cm}} / L_{\mathrm{B}}\right)$. Radio-quiet objects show values of $R$ concentrated between $0.1-1$, while in radio-loud sources the $R$ values range from 10 to 100 (Kellerman et al. 1989), so that the boundary between the two classes is normally defined at $R=10$ (Visnovsky et al. 1992; Kellerman et al. 1994). Another criterion introduced by Miller et al. (1990) is based on the separation between the two classes in the radio luminosity $\left(P_{6 \mathrm{~cm}} \sim 10^{25} \mathrm{~W} \mathrm{~Hz}^{-1} \mathrm{sr}^{-1}\right)$. The existence of the bimodality in the AGN population has been recently questioned and ascribed to sample selection effects, i.e., in deep radio and optical surveys no bimodality is observed in the distribution of the radio loudness parameter (White et al. 2000; Hewett et al. 2001; Cirasuolo et al. 2003). However, on the base of a sample of
10000 objects selected from SDSS and FIRST, Ivezić et al. (2004, 2002) still found a bimodal distribution of $R$.

Seyfert galaxies and low luminosity AGN (LLAGN ${ }^{1}$ ) have been surveyed by VLA and VLBI observatories (Ho \& Ulvestad 2001; Nagar et al. 2002). Ho \& Ulvestad (2001) have shown that $85 \%$ of the Seyfert nuclei are detected at $6 \mathrm{~cm}$. These authors found a wide range of radio powers and morphologies of the radio emission typical of that of a compact core (either unresolved or slightly resolved), occasionally accompanied by elongated, jet-like features. Similarly, Nagar et al. (2002) have detected a high incidence of pc-scale radio cores and sub-parsec jets in LLAGN. However, the physics of jets in radio-quiet sources is still unclear. Henri \& Petrucci (1997) and Malzac et al. (1998) suggested that the initial part of a jet can produce relativistic particles illuminating the disk, with the jet having either bulk relativistic motion or containing very energetic particles. Alternatively, the bulk velocities could be sub-relativistic in a sort of "aborted jet", which also produces emission at X-ray frequencies (Ghisellini et al. 2004). This idea would be an alternative to the disk/hot corona model that is commonly invoked to explain the observed X-ray emission in radio-quiet AGN (Haardt \& Maraschi 1991).

The combination of data on both X-ray and radio frequencies offers the opportunity to investigate the relationship between the physical source of the X-ray emission, likely due to the disk-corona system in Seyfert galaxies, and the radio source.

\footnotetext{
1 As LLAGN we refer to low luminosity Seyfert galaxies, LINERs, and "transition nuclei" with spectra intermediate between those of LINERs and HII regions.
} 
Monochromatic soft X-ray and radio luminosities have been correlated in radio-quiet and radio-loud quasars (Salvato et al. 2004; Brinkmann et al. 2000; Canosa et al. 1999), confirming the idea that the radio emission originates in a compact nuclear source directly associated with the central engine. Recently, Falcke et al. (2004) and Merloni et al. (2003) have combined the X-ray, radio luminosity, and black hole mass estimates and derived the so-called fundamental plane of super-massive black hole activity. Falcke et al. (2004) suggested that the radio through X-ray emission of LLAGN is attributed to synchrotron emission from a relativistic jet, similarly to the scenario proposed for X-ray binaries (XRBs) in their low/hard state. Moreover, the slope found for the X-ray and radio relation in their work is consistent with that observed in stellar black hole systems (Gallo et al. 2003). On the other hand, the fitting results by Merloni et al. (2003) require a radiatively inefficient accretion flow (Narayan \& Yi 1994; Abramowicz 1997) as the origin for X-ray emission, and a relativistic jet as the origin for the radio emission. Since the radiatively inefficient accretion flow models apply for low Eddington ratios $\left(L / L_{\text {Edd }} \leq 10^{-2}\right)$, this theoretical view can be suitable for LLAGN, which are known to accrete at very low Eddington ratios. However, several pieces of evidence have been accumulated showing that most of the low luminosity Seyfert galaxies and LLAGN share characteristics in common with their more luminous counterparts, such as broad $\mathrm{H}_{\alpha}$ emission lines and emission line ratios characteristic of powerful AGN in the optical band (Ho et al. 1997a), and spectral slopes and FeK line features typical of luminous AGN in the X-ray band (e.g., Cappi et al. 2005; Ho et al. 2001); altogether these point to similar emission processes and to radiatively efficient accretion mechanisms.

Indeed, a homogeneous X-ray study of a well-defined sample of nearby Seyfert galaxies recently performed has confirmed a continuity between the properties of low luminosity Seyfert galaxies and luminous AGN (Cappi et al. 2006; Panessa et al. 2006). We have shown that the distributions of the spectral parameters (such as the continuum slope, the energy of the FeK line, the absorbing column density, etc.), in particular for type 1 objects, are found to be within the range of values observed in luminous AGN. In Panessa et al. (2006) we have also investigated the X-ray, optical, and black hole mass properties for a sample of Seyfert galaxies with luminosities spanning from $10^{37} \mathrm{erg} \mathrm{s}^{-1}$ up to $10^{43} \mathrm{erg} \mathrm{s}^{-1}$. Our study is consistent with the idea that Seyfert nuclei are a scaled-down version of luminous AGN. The strong correlations found between X-ray and optical emission lines over nearly 8 orders of magnitude suggest that the X-ray source and the accretion mechanism responsible for the production of UV photons are strongly related, similarly to quasars and independently of their nuclear activity level, and, therefore, consistently with radiative efficient accretion. In this paper, we have continued the investigation of the multiwavelength properties of the Palomar Seyfert sample, exploiting the availability of high spatial resolution data both at X-ray and radio frequencies and correlating them for the first time.

A description of the sample is given in Sect. 2; the X-ray versus radio at $20 \mathrm{~cm}, 6 \mathrm{~cm}$, and $2 \mathrm{~cm}$ correlation for Seyfert galaxies is presented in Sect. 3; the relation between X-ray and $6 \mathrm{~cm}$ radio luminosities is discussed in Sect. 4, where the Palomar Seyfert sample is also compared to a sample of low luminosity radio galaxies; in Sect. 5 we investigated the properties of the radio loudness parameter, and in Sect. 6 we explored its relationship with the black hole mass and the Eddington ratio. Finally, results are discussed and summarized in Sect. 7. All distancedependent quantities were transformed to our adopted cosmological parameters of $H_{0}=75 \mathrm{~km} \mathrm{~s}^{-1} \mathrm{Mpc}^{-1}$ and $q_{0}=0.5$.

\section{The sample}

The Seyfert sample studied here has been presented in a companion paper (Panessa et al. 2006). It comprises 47 out of 60 Seyfert galaxies from the Palomar optical spectroscopic survey of nearby galaxies (Ho et al. 1995) for which X-ray data are available. The sources are classified as type 2 (34 out of 60), type 1 (13 out of 60), and "mixed" Seyfert galaxies (8), according to their position in the optical emission line diagnostic diagrams. The "mixed" Seyferts are found near the boundary between Seyfert and LINER, HII or transition classification, resulting in a double classification (e.g., S2/T2, L2/S2, H/S2, etc.). See Panessa et al. (2006) for a more detailed description of the sample.

\section{The $L_{X}$ versus $L_{R}$ correlation in the Palomar Seyfert sample}

The Palomar Seyfert sample has the merit of having very accurate emission measurements in most of the spectral wavelengths. In this section, we explore the relationship between the X-ray and radio luminosities, combining nuclear X-ray and core radio data obtained in recent surveys for the first time (Cappi et al. 2006; Panessa et al. 2006; Ho \& Ulvestad 2001; Nagar et al. 2002). In Panessa et al. (2006), we have presented an X-ray study of this sample, using the most recent Chandra, XMM-Newton, and, only in a few cases, ASCA observations. The nuclear 2-10 keV X-ray luminosities, obtained with minimal contamination by off-nuclear sources and diffuse emission, have been corrected for galactic and intrinsic absorption, the latter by using the measured X-ray column densities. Among type 2 Seyfert galaxies, a sub-sample of 11 candidate Compton thick sources ( $>30 \%$ of type 2 Seyferts) has been found. Since most of the intrinsic luminosity of Compton thick sources is obscured from our line of sight and the intrinsic absorption is not measurable by data below $10 \mathrm{keV}$, we applied a correction factor to the observed luminosities of these objects. For a detailed description of the treatment of Compton thick candidate luminosities we refer to Cappi et al. (2006) and Panessa et al. (2006).

Ho \& Ulvestad (2001) have undertaken a radio continuum survey of 52 Palomar Seyfert galaxies using the Very Large Array (VLA). The observations were made at $6 \mathrm{~cm}$ and at $20 \mathrm{~cm}$ with an angular resolution of $\sim 1^{\prime \prime}$. Nearly $47 \%$ of the Seyfert galaxies from the Palomar sample have also been detected by a VLA survey at $2 \mathrm{~cm}$, performed with a resolution of $0.15^{\prime \prime}$ (Nagar et al. 2005). Moreover, we have included data at $6 \mathrm{~cm}$ from Filho et al. (2002) for a group of four "mixed" sources, NGC 3489, NGC 3627, NGC 6482, and NGC 6503, not included in the Ho \& Ulvestad (2001) sample.

In Fig. 1, we plot the absorption-corrected 2-10 keV luminosity versus the core radio luminosity at $20 \mathrm{~cm}$ (left), $6 \mathrm{~cm}$ (center), and $2 \mathrm{~cm}$ (right). In each case, we have performed a partial Kendall $\tau$ correlation test, which computes partial correlation coefficient and significance for censored data using three variables, where we took the distance as the third variable (Akritas $\&$ Siebert 1996). The X-ray versus radio luminosity correlation is highly significant at all radio frequencies considered (with a probability greater than $99.9 \%$ ). In Table 1 we also report the Spearman's rho correlation coefficient for comparison. We characterize the above correlations by using the Schmitt method, which treats censored data in both variables (Schmitt et al. 1985, the algorithm is implemented in the ASURV package, Isobe et al. 1986). We report the Schmitt's slope and intercept in Table 1 as well. 

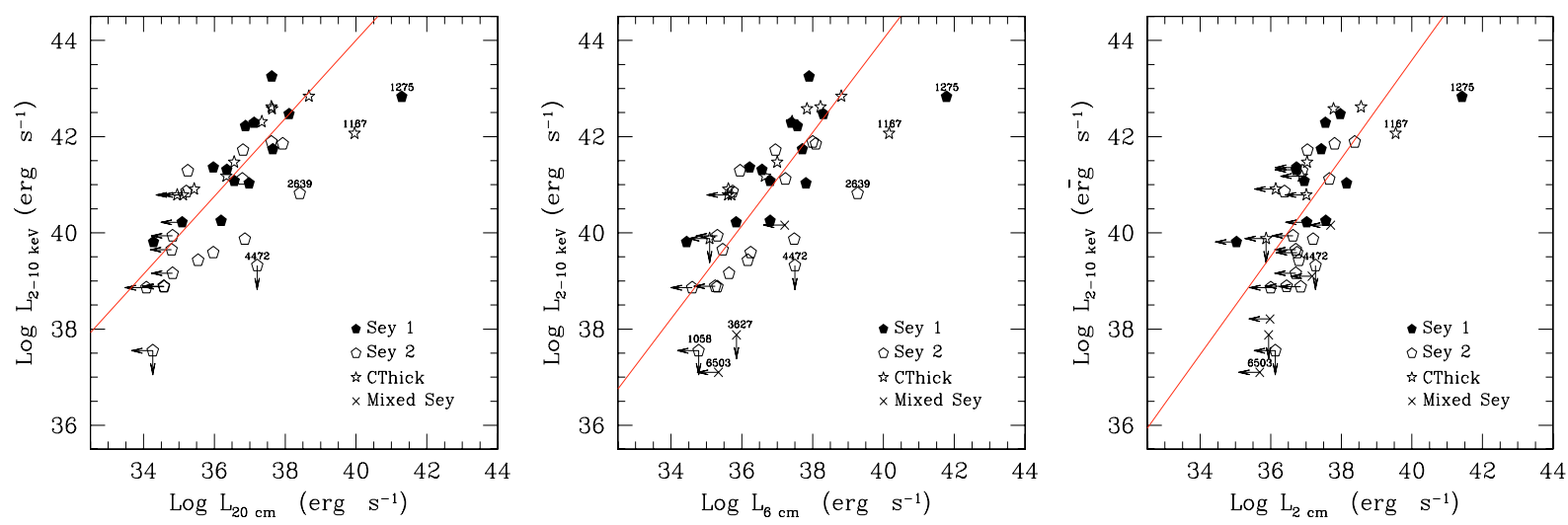

Fig. 1. Intrinsic 2-10 keV luminosity versus core radio luminosity at $20 \mathrm{~cm}$ (left), $6 \mathrm{~cm}$ (center), and $2 \mathrm{~cm}$ (right). Radio data at $20 \mathrm{~cm}$ and $6 \mathrm{~cm}$ are taken from Ho \& Ulvestad (2001), and at $2 \mathrm{~cm}$ are from Nagar et al. (2005). Type 1 objects are plotted as filled polygons, type 2 as empty polygons, "mixed Seyfert" objects as crosses, and Compton thick candidates as stars. The NGC names of a group of radio-loud Seyfert galaxies has been highlighted.

Table 1. Correlation statistics in luminosities.

\begin{tabular}{|c|c|c|c|c|c|c|c|c|c|c|}
\hline $\begin{array}{l}\text { Variables } \\
\text { (1) }\end{array}$ & $\begin{array}{l}N \\
(2) \\
\end{array}$ & $\begin{array}{c}X(\mathrm{ul}) \\
(3)\end{array}$ & $\begin{array}{c}Y(\mathrm{ul}) \\
(4)\end{array}$ & $\begin{array}{c}X Y(\mathrm{ul}) \\
(5)\end{array}$ & $\begin{array}{c}\text { Spearman } \\
\text { (6) }\end{array}$ & $\begin{array}{c}\tau \\
(7) \\
\end{array}$ & $\begin{array}{c}\sigma \\
(8) \\
\end{array}$ & $\begin{array}{c}\text { Prob. } \\
(9)\end{array}$ & $\begin{array}{c}a \\
(10)\end{array}$ & $\begin{array}{c}b \\
(11)\end{array}$ \\
\hline \multicolumn{11}{|l|}{ Seyfert galaxies } \\
\hline \multirow{2}{*}{$\log L_{\mathrm{X}}$ Vs. $\log L_{20 \mathrm{~cm}}$} & 41 & 2 & 2 & 8 & 0.79 & 0.50 & 0.10 & $7.15 \mathrm{E}-07$ & $0.62 \pm 0.04$ & $18.17 \pm 1.46$ \\
\hline & $37^{a}$ & 8 & 1 & 2 & 0.87 & & & & $0.81 \pm 0.02$ & $11.60 \pm 0.72$ \\
\hline \multirow[t]{2}{*}{$\log L_{\mathrm{X}}$ Vs. $\log L_{6 \mathrm{~cm}}$} & 45 & 6 & 2 & 1 & 0.78 & 0.43 & 0.09 & $<1.0 \mathrm{E}-08$ & $0.71 \pm 0.01$ & $14.62 \pm 0.28$ \\
\hline & $40^{b}$ & 5 & 1 & 1 & 0.84 & & & & $0.97 \pm 0.01$ & $5.23 \pm 0.28$ \\
\hline \multirow[t]{2}{*}{$\log L_{\mathrm{X}}$ vs. $\log L_{2 \mathrm{~cm}}$} & 41 & 15 & 2 & 2 & 0.68 & 0.36 & 0.07 & $7.15 \mathrm{E}-07$ & $0.80 \pm 0.05$ & $10.77 \pm 1.87$ \\
\hline & $37^{c}$ & 14 & 1 & 2 & 0.67 & & & & $1.02 \pm 0.03$ & $2.79 \pm 0.96$ \\
\hline \multicolumn{11}{|c|}{ Low-luminosity radio galaxies } \\
\hline $\log L_{X}$ VS. $\log L_{6 \mathrm{~cm}}$ & 33 & 6 & 5 & 1 & 0.76 & 0.42 & 0.10 & $1.67 \mathrm{E}-05$ & $0.97 \pm 0.02$ & $2.42 \pm 0.92$ \\
\hline
\end{tabular}

Notes: Statistical properties of the 2-10 keV X-ray luminosity versus radio luminosities at $20 \mathrm{~cm}, 6 \mathrm{~cm}$, and $2 \mathrm{~cm}$; Col. (1): Number of sources; Cols. (2)-(4): Number of upper limits in variable $X, Y$, and both; Col. (5) Spearman's rho correlation coefficient; Cols. (6)-(8) Kendall's $\tau$ correlation coefficient, the square root of the variance, $\sigma$, and the associated probability $P$ for accepting the null hypothesis that there is no correlation; Cols. (9), (10): Correlation coefficient of the best-fit linear regression line calculated using Schmitt binned linear regression method, $Y=a \times X+$ $b$. Radio-loud sources excluded: ${ }^{a}$ NGC 1275, NGC 1167, NGC 2639, and NGC 4472; ${ }^{b}$ NGC 1275, NGC 1167, NGC 2639, NGC 4472, and NGC 6503; ${ }^{c}$ NGC 1275, NGC 1167, NGC 4472, and NGC 6503.

The X-ray versus radio luminosity correlations in Fig. 1 show a group of outliers, i.e., sources that present an excess in the radio emission with respect to the average $\mathrm{X}$-ray/radio ratio shown by the sample. Interestingly, these sources have been classified in previous works as radio-loud objects, as we briefly discuss in the following. The type 1 Seyfert having the highest radio luminosity in each plot is NGC 1275; this is a radio-loud source (3C 84) having well-known jets studied in detail (Ho \& Ulvestad 2001, and references therein). NGC 1167 (a likely Compton thick candidate) is a well-known radio source $(4 \mathrm{C}+34.09)$, and it has been extensively studied at radio frequencies (Komossa et al. 1999). NGC 2639 is a radio-loud galaxy with an extended radio morphology. When observed at higher spatial resolution (VLBI), NGC 2639 is found to be one of the rare examples of a radio-emitting spiral galaxy with a VLBI core source (Hummel et al. 1982). Finally, NGC 4472, an early-type galaxy, is also a radio-loud source (Caon et al. 1994).

Another small group of outliers is visible in the $L_{\mathrm{X}}$ vs. $L_{6} \mathrm{~cm}$ plot (central panel of Fig. 1), at X-ray luminosities lower than $10^{38} \mathrm{erg} \mathrm{s}^{-1}$. Two out of these three sources are classified as "mixed Seyfert", i.e., NGC 3627 and NGC 6503, while NGC 1058, which is a type 2 Seyfert galaxy, has an upper limit to the X-ray luminosity. As already discussed in Panessa et al. (2006), these sources show peculiar behavior and their emission could have a starburst origin rather than an AGN nature.

In Table 1, we have reported the best-fit linear regression line obtained at each radio frequency either by including the above radio-loud sources and by excluding them; the latter is the fit plotted in Fig. 1. Note that the slope of the regression line becomes steeper when considering higher radio frequencies, from $20 \mathrm{~cm}$ to $6 \mathrm{~cm}$ and $2 \mathrm{~cm}$. The observed correlations suggest that the X-ray and radio emission mechanisms are strongly related in Seyfert galaxies, as further discussed in the next section.

\section{The $L_{x}$ versus $L_{6 \mathrm{~cm}}$ correlation}

In this section we focus on the $L_{2-10} \mathrm{keV}$ versus $L_{6} \mathrm{~cm}$ correlation and compare the Palomar Seyfert galaxy sample with a sample of low luminosity radio galaxies taken from two different catalogues. Balmaverde \& Capetti (2006) have selected a sample of 116 early-type galaxies (morphological Hubble type $T \leq-1$ ) detected in VLA surveys limited in flux at $\sim 1 \mathrm{mJy}$. Using HST data for 65 out of 116 sources, the sources have been separated into "core" and "power-law" galaxies according to their nuclear brightness profiles. Here, we focus our study on these "core" galaxies that have been optically classified as type 1 Seyferts, type 2 Seyferts, and LINERs, when an optical classification is 

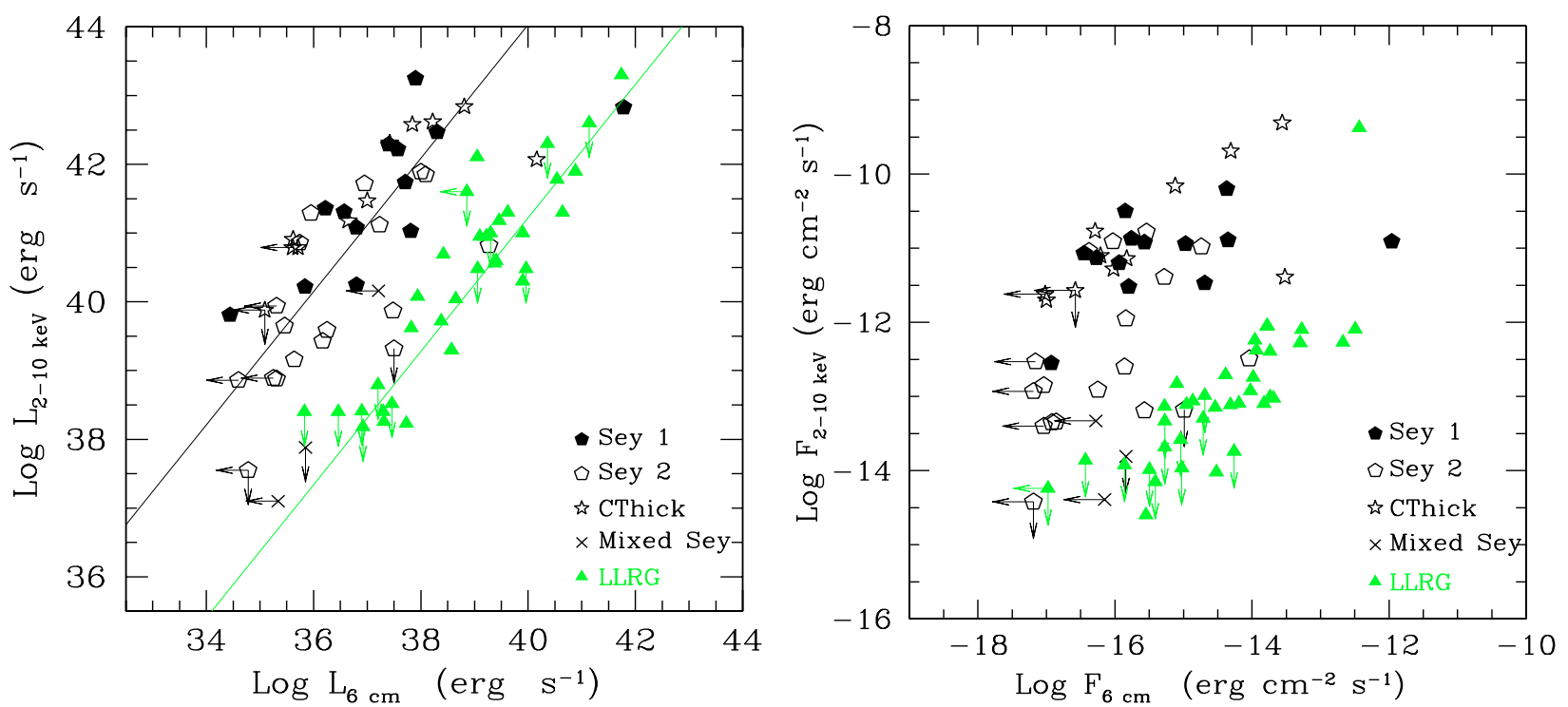

Fig. 2. Left panel: intrinsic $2-10 \mathrm{keV}$ luminosity versus core radio luminosity at $6 \mathrm{~cm}$. Seyfert galaxies are plotted as polygons. Low-luminosity radio galaxies are plotted as solid triangles (Balmaverde \& Capetti 2006). Seyferts' and LLRGs' best-fit linear regression lines obtained using the Schmitt method have been plotted. Right panel: same plot of left panel in flux-flux space.

available. The radio maps of the 29 "core" radio galaxies indicate collimated outflows, which suggest that miniature radiogalaxies are probably residing in these sources (Balmaverde \& Capetti 2006). Nuclear Chandra X-ray luminosities are available for 20 out of 29 sources.

In addition, we also considered a sample of 16 low luminosity radio galaxies from the $3 \mathrm{C} / \mathrm{FRI}$ sample (Chiaberge et al. 2005), for which Chandra X-ray luminosities are reported in Balmaverde \& Capetti (2006). The optical classifications of the sources belonging to this sample span from Seyfert galaxies (both of type 1 and type 2) to LINERs. Note that NGC 4261, NGC 4374, and NGC 4486 are both in the "core" radio galaxy sample and in the 3C/FRI sample, so when computing the bestfit regression lines we considered them only once. Also note that the type 1 Seyfert galaxy NGC 1275 (3C84) also belongs to the 3C/FRI sample, while the type 1.9 Seyfert NGC 4168 (UGC 7203) is also included in the "core" galaxy sample. Here we treat the core galaxy and the 3C/FRI galaxy samples as a unique sample of low-luminosity radio galaxies (LLRGs).

In Fig. 2 (left panel) we plot the nuclear 2-10 keV luminosity versus the core radio luminosity at $6 \mathrm{~cm}$, for the Palomar Seyfert sample and the LLRGs. The LLRGs best-fit regression line has been obtained using the Schmitt method, considering the censored data as reported in Table 1. Our best-fit is in agreement, within errors, with that reported in Balmaverde \& Capetti (2006) obtained by excluding upper limits (with a slope of $1.02 \pm 0.10$ ). Interestingly, the group of Seyfert galaxies with radio excess emission discussed in the previous section, lay in the LLRGs correlation. The main result that emerges from this analysis is that the radio and $X$-ray luminosities in Seyfert galaxies and LLRGs are significantly correlated; in addition, both samples show the same regression slope, shifted by nearly three orders of magnitude:

- Seyferts: $\log L_{\mathrm{X}}=(0.97 \pm 0.01) \log L_{6 \mathrm{~cm}}+(5.23 \pm 0.28)$

- LLRGs: $\log L_{X}=(0.97 \pm 0.02) \log L_{6} \mathrm{~cm}+(2.42 \pm 0.92)$.

The flux-flux plot in Fig. 2 (right panel) shows a larger scatter; however, the correlation remains statistically significant $(>99.9 \%)$, meaning that the two correlations are not driven by distance effects, as is also demonstrated by the Kendall's $\tau$ test result (Table 1 ). However, it must be taken into account that selection effects are probably introduced in the Seyfert and LLRG samples. The Seyfert galaxy sample has been derived from the optical flux-limited sample of the Palomar survey (Ho et al. 1995). Originally, the survey contained a sample of 60 Seyfert galaxies, which was indeed complete. We have X-ray data for 47 out of 60 sources, thus we are probably introducing a slight bias toward high luminosity Seyfert nuclei. The missing sources should have X-ray luminosities below $\log L_{2-10 \mathrm{keV}} \sim$ $40.5 \mathrm{erg} \mathrm{s}^{-1}$ (see Panessa et al. 2006). A different selection procedure has been introduced when building the core galaxy sample by Balmaverde \& Capetti (2006). These are early-type galaxies, initially selected from a flux-limited VLA survey, which also has HST and Chandra data available. This sample is not complete in any sense and is probably biased towards high luminosity nuclei. Therefore, in both Seyfert and LLRG samples, the bias at low luminosities could affect our results, but only slightly, since the slopes of the correlations are calibrated for over 8 orders of magnitude, while the lower luminosity sources should account for only one or at most two orders of magnitude in the correlations. In any case, this does not affect the observed dichotomy between Seyfert galaxies and LLRGs, i.e., putative objects with an X-ray and a radio luminosity potentially populating the gap between the two correlations are within the selection criteria of the two samples and therefore, if existing, they should have been detected in both cases. However, understanding the possible effects introduced by comparing a radio and an optical flux-limited sample is not a simple task. Complete and bias-free samples are needed to overcome the present limitation.

In the literature, X-ray data, taken with ROSAT, have been correlated with $6 \mathrm{~cm}$ radio core luminosities for a sample of low-power radio galaxies (Canosa et al. 1999). Interestingly, despite the use of $1 \mathrm{keV}$ luminosity instead of the $2-10 \mathrm{keV}$ one as in our case, the best-fit Schmitt slope of the regression line found by these authors is consistent within errors with our findings (slope $=1.15_{-0.23}^{+0.24}$ ). Brinkmann et al. (2000) correlated the ROSAT $2 \mathrm{keV}$ monochromatic luminosity and the $6 \mathrm{~cm}$ luminosity for a sample of radio-loud and radio-quiet galaxies, derived from a cross-correlation of the ROSAT All Sky Survey and the VLA $20 \mathrm{~cm}$ FIRST catalogue. The slope of the bestfit regression line of radio-quiet galaxies turned out to be steeper 
than those obtained for radio-loud objects $(1.012 \pm 0.083$ and $0.483 \pm 0.049$, respectively). Note that this sample is biased toward high luminosity sources, i.e., no radio-loud galaxies with $\log L_{\mathrm{R}} \leq 40.5 \mathrm{erg} \mathrm{s}^{-1}$ are present, and the regression line is calibrated only over 4 orders of magnitude. A soft X-ray versus radio (at $1.94 \mathrm{GHz}$ ) luminosity correlation has also been observed in a sample of 93 AGN selected from the ROSAT Bright Survey (Salvato et al. 2004), with a correlation slope that is in agreement with that found for XRBs, $L_{\mathrm{R}} \propto L_{\mathrm{X}}^{0.7}$ (Gallo et al. 2003).

Here, the X-ray versus radio luminosity correlations found are valid for nearly eight orders of magnitude in both Seyfert and LLRG samples implying that (i) the X-ray and the radio source emission are strongly coupled down to very low luminosities, and (ii) they follow a similar correlation slope in the two samples, suggesting either a common set of physical mechanisms producing the emission or a combination of different mechanisms that end up producing a similar correlation slope. There are severe limitations to our knowledge of the X-ray and radio source emission both in radio-loud and radio-quiet AGN. For example, the physical origin of X-ray emission in radio-loud AGN is a largely debated topic. On the one hand, it has been proposed that the nuclear X-ray emission is originated at the base of a parsec-scale radio jet (e.g., Fabbiano et al. 1984). The flatness of the X-ray slope correlates with radio core dominance for radio-loud quasars, suggesting that a component of the X-ray emission is relativistically beamed (Zamorani et al. 1986; Shastri et al. 1993). Moreover, their multi-wavelength emission is interpreted in terms of synchrotron and inverse Compton jet models only (Chiaberge et al. 2003; Pellegrini et al. 2003). Finally, the correlations between the radio core power and X-ray luminosities (Worrall 1997; Canosa et al. 1999; Brinkmann et al. 2001) observed for radio galaxies suggest that the X-ray spectrum is produced by Compton up-scattering of the radio synchrotron photons from the relativistic jet. On the other hand, the detection of broadened FeK lines and short-term variability (Gliozzi et al. 2004) support the idea that the emission originates in an accretion flow. Evidence in favor of the presence of the two components has been found in a few sources (Evans et al. 2004; Zezas et al. 2004; Sambruna et al. 2006).

Balmaverde \& Capetti (2006) have shown that LLRGs in their sample are genuine active nuclei that host a radio-loud core. They found correlations between optical, X-ray, and radio luminosities suggesting a common non-thermal origin of the nuclear emission. They suggest that, given the low values of the Eddington ratios obtained, the radiative output of these sources is most likely dominated by the jet, with only a small fraction due to the accretion process.

In the case of Seyfert galaxies, the most accepted physical scenario for their X-ray spectra assumes that the emission is produced by a disk-corona system, in which UV-soft photons from the accretion disk are comptonized and up-scattered into the hard $\mathrm{X}$-ray band by a hot corona above the accretion disk (Haardt \& Maraschi 1991). In agreement with this theoretical picture, $\mathrm{X}$-ray studies of Seyfert galaxies have shown that their intrinsic X-ray spectral and emission properties are similar to those of more luminous AGN, including objects with very low luminosities down to $L_{\mathrm{X}} \sim 10^{38} \mathrm{erg} \mathrm{s}^{-1}$ (Ho et al. 2001; Terashima \& Wilson 2003; Cappi et al. 2006; Panessa et al. 2006). For a given $\mathrm{X}$-ray luminosity, the Palomar Seyfert galaxies are three orders of magnitude weaker in the radio band with respect to LLRGs. The origin of the radio emission and the physics of jets in Seyfert galaxies and LLAGN is not yet understood. The radio parsec and sub-parsec scale study of a few LLAGN indicates that the radio emission is due to a synchrotron process from the base of a radio jet, as in more luminous radio-loud AGN (Giroletti et al. 2005; Nagar et al. 2005). Conversely, Bicknell (2002) suggests that there are major differences between Seyfert and radio galaxies jets: Seyfert's jets are thermally dominated with sub-relativistic speeds, while radio galaxies' jets are relativistic electron/positron flows. Another possible scenario has been proposed by Ghisellini et al. (2004) to explain radio emission in Seyfert galaxies, such as the presence of "aborted jets" also responsible for the emission in X-rays. The latter theory could explain the correlation found between the radio and X-ray luminosity in radio-quiet AGN. The correlations found here can be used, when compared to theoretical predictions, to constrain the physical parameters and improve our knowledge on how the disk-jet connection works in AGN.

\section{The radio-loudness}

Both the existence of a real dichotomy between radio-loud and radio-quiet AGN (not simply due to sample selection effects) and its origin are still a matter of debate. Seyfert galaxies are traditionally considered to be radio-quiet objects, because of their low radio power and the small radio-to-optical ratio $(R<$ 10). However, Ho \& Peng (2001) have shown that, by considering their nuclear luminosities, most Seyfert galaxies are instead radio-loud AGN. In these sources, the host galaxy emission plays an important role in the determination of the photometric measurements, sometimes leading to a large overestimate of the optical flux. Ho \& Peng (2001) measured the optical luminosities from HST images, where the high spatial resolution allows them to eliminate the contribution from stellar light to the overall emission. They recomputed the radio and optical fluxes for a sub-sample of the Palomar Seyfert galaxies (mostly type 1-1.5) and found that most of the objects analyzed are indeed radio-loud, having their radio-loudness parameter $R$ higher than the classical boundary value set at $R=10$ (Kellermann et al. 1994, and references therein). Note, however, that by instead using the luminosity criterion, $P_{6 \mathrm{~cm}} \sim 10^{25} \mathrm{~W} \mathrm{~Hz}^{-1} \mathrm{sr}^{-1}$, only NGC 1275 turns out to be radio-loud in our sample.

We calculate the $R$ parameter, $L_{v}(6 \mathrm{~cm}) / L_{v}(\mathrm{~B})$, as shown in the distribution of Fig. 3 (right panel). The optical data for LLRGs have been taken from Balmaverde \& Capetti (2006), while $R$ values for a sub-sample of type 1-1.9 Palomar Seyfert galaxies are taken from Ho \& Peng (2001). The mean value for LLRGs is $3.74 \pm 0.11$ compared to $1.93 \pm 0.26$ for the Seyfert galaxies and the two distributions do not belong to the same parent population (KS probability of $1.08 \times 10^{-8}$ ).

Recently, Terashima \& Wilson (2003) have introduced a new definition of the radio loudness parameter by comparing the $6 \mathrm{~cm}$ radio luminosity to the $2-10 \mathrm{keV}$ luminosity, $R_{\mathrm{X}} \equiv$ $L_{v}(6 \mathrm{~cm}) / L(2-10 \mathrm{keV})$. The use of the X-ray luminosity with respect to the optical one should largely avoid extinction problems that normally occur in the optical band and that could cause an overestimation of $R$. We therefore calculated the $R_{\mathrm{X}}$ parameter for both the Seyfert galaxies and the LLRG sample (see the distributions of the two samples in Fig. 3, left panel). A Kolomogorov-Smirnov test, computed by excluding upper limits, results in a probability of $3.5 \times 10^{-13}$ that the two samples are drawn from the same parent population. The mean $\log R_{\mathrm{X}}$ value of LLRGs is $-1.40 \pm 0.11$, compared to $-3.64 \pm$ 0.16 of Seyfert galaxies.

The two classes of sources show clearly different distributions in both the $R_{\mathrm{X}}$ and the $R$ parameters, as is also clear in Fig. 4, where we plot the $\log R$ versus $\log R_{\mathrm{X}}$. We have compared the Seyferts and LLRG cumulative distributions for the 

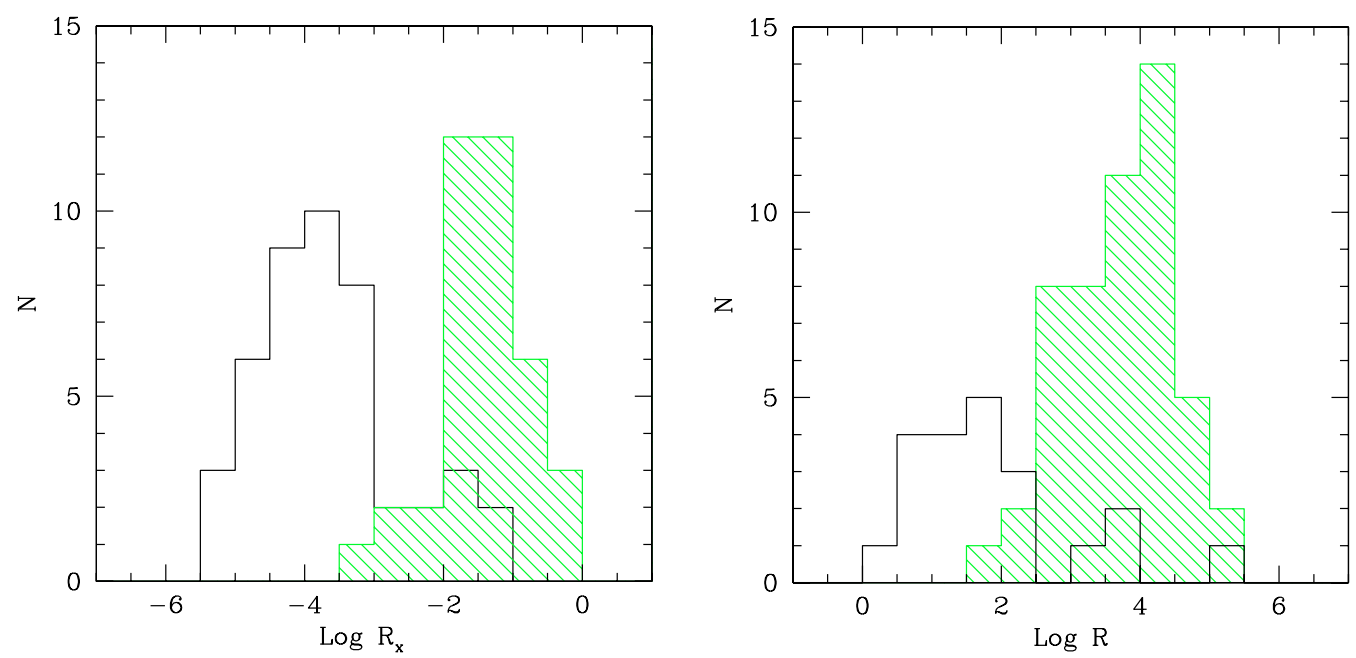

Fig. 3. Left panel: distributions of the $R_{\mathrm{X}} \equiv L_{\nu}(6 \mathrm{~cm}) / L(2-10 \mathrm{keV})$ parameter for the Seyferts sample (left) and LLRG sample (right, shaded histogram). Right panel: distributions of the $R \equiv L_{\nu}(6 \mathrm{~cm}) / L_{\nu}(\mathrm{B})$.

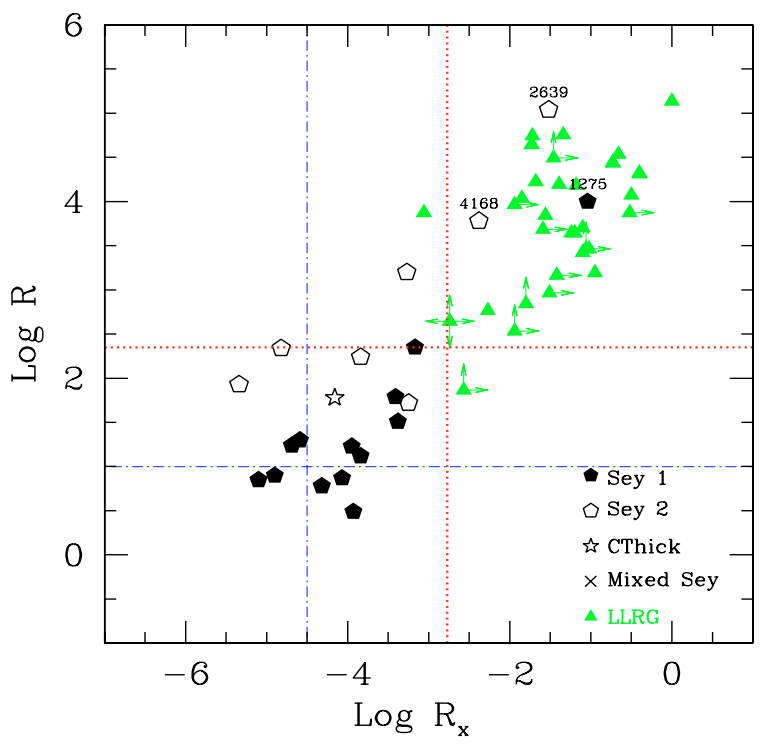

Fig. 4. Relation between the radio-loudness parameters $R \equiv$ $L_{v}(6 \mathrm{~cm}) / L_{v}(\mathrm{~B})$ and $R_{\mathrm{X}} \equiv L_{v}(6 \mathrm{~cm}) / L(2-10 \mathrm{keV})$ for the Seyferts sample (polygons) and the LLRG sample (triangles). Horizontal and vertical lines represent the values where the maximum difference between the $R_{\mathrm{X}}$ and $R$ parameter distributions of Seyfert galaxies and LLRGs occurs. Note that 3C028 has an upper limit at all frequency measurements, therefore the radio-loudness ratios cannot be determined.

$R_{\mathrm{X}}$ parameter by using the KS test and found that the maximum separation between the two distributions $(D=0.81)$ is located at $R_{\mathrm{X}}=-2.755 \pm 0.015$. In the case of the $R$ parameter, the maximum distance $(D=0.77)$ is found at $R=-2.400 \pm 0.050$. We plotted the two boundaries as vertical and horizontal dotted lines in Fig. 4.

Terashima \& Wilson (2003) have compared the $R$ and the $R_{\mathrm{X}}$ radio-loudness parameters for a sample of LLAGN, Seyfert galaxies, and PG quasars, deriving a relation between the two variables $\left(\log R=0.88 \log R_{\mathrm{X}}+5.0\right)$. We also calculated the best-fit linear regression line: $\log R=0.91 \pm 0.06 \log R_{\mathrm{X}}+$ $5.28 \pm 0.18$. Within errors, the latter is consistent with that found by Terashima \& Wilson (2003). These authors used that relationship to derive the boundary between the radio-loud and radio-quiet objects in $R_{\mathrm{X}}$, i.e., by fixing $R=10$, they obtained $\log R_{\mathrm{X}}=-4.5$. According to the above boundaries, most Seyfert galaxies should be considered as radio-loud AGN, as well as all the LLRGs. However, if we take into account the radio-loud nature of LLRGs (Balmaverde \& Capetti 2006) and consider the different radio powers of Seyfert galaxies with respect to LLRGs, we could redefine the boundaries between radio-loud and radio-quiet AGN as above: $R_{\mathrm{X}}=-2.755 \pm 0.015$ and $R=$ $-2.400 \pm 0.050$. The validity of such limits should be tested in the future with larger samples of radio-quiet and radio-loud AGN. These different boundaries found for low luminosity active nuclei with respect to those of luminous AGN could suggest a dependence of $R$ and $R_{\mathrm{X}}$ with luminosity. However, as shown in Fig. 5 where we plot the radio loudness parameters, $R_{\mathrm{X}}$ (left panel) and $R$ (right panel), versus the $\mathrm{X}$-ray luminosity, there is no evidence of such a trend within the low luminosities spanned by our sample.

\section{Radio-loudness versus $M_{\mathrm{BH}}$ and Eddington ratio}

Several recent works have attempted to interpret the occurrence of radio-loud nuclei in the center of only a few percent of AGN, as related to the host galaxy properties. For example, it has been observed that spiral galaxies preferentially harbor radio-quiet $\mathrm{AGN}$, while early-type galaxies host both radio-quiet and radio-loud AGN (Hutchings 1983). Actually, LLRGs have been selected to be early-type galaxies while only a few Seyfert galaxies are hosted by elliptical host galaxies (e.g., NGC 3608, NGC 4168, NGC 4472, and NGC 6482). Moreover, Dunlop et al. (2003) have found that radio-loud AGN are associated with the most massive black hole hosts, providing a natural explanation for why radio-quiet AGN outnumber radio-loud AGN, and suggesting that the radio output is a strong function of the black hole mass.

In Fig. 6 we present the black hole mass distribution for the sample of Seyfert galaxies (top) and LLRGs (bottom). The $M_{\mathrm{BH}}$ estimates for the Seyfert galaxies have been taken from the literature, where they were obtained using a variety of methods (Panessa et al. 2006). Palomar Seyfert $M_{\mathrm{BH}}$ are distributed from $\sim 10^{5}$ to $10^{8} M_{\odot}$ with a peak at $10^{7-8} M_{\odot}$. The $M_{\mathrm{BH}}$ distribution of LLRGs has already been shown in Capetti \& Balmaverde (2006): core galaxies and low luminosity radio galaxies in their sample show a similar $M_{\mathrm{BH}}$ distribution and a narrow range of 

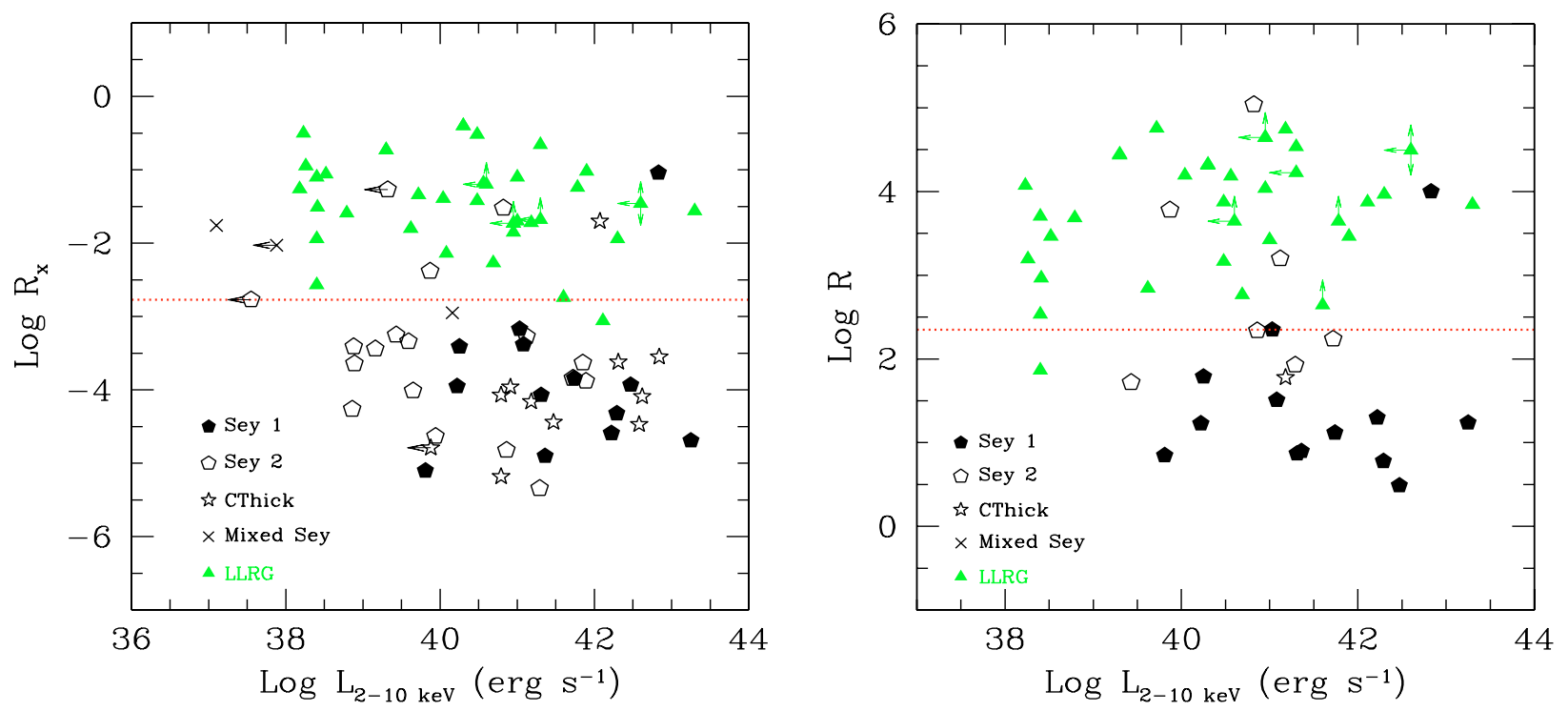

Fig. 5. Left panel: $R_{\mathrm{X}} \equiv L_{v}(6 \mathrm{~cm}) / L(2-10 \mathrm{keV})$ and $R \equiv L_{\nu}(6 \mathrm{~cm}) / L_{v}(\mathrm{~B})$ versus $\log L(2-10 \mathrm{keV})\left(\mathrm{erg} \mathrm{s}^{-1}\right)$, left and right panel respectively. The lines representing the values where the maximum difference between the are plotted respectively in the left and right panel.

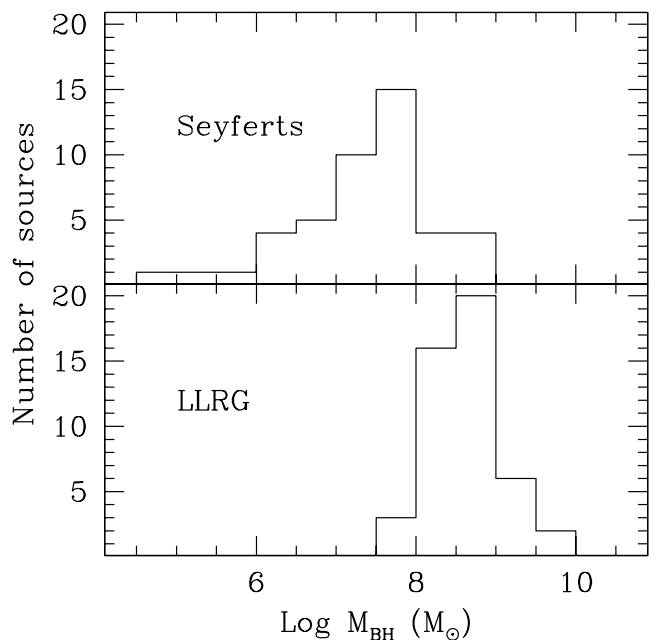

Fig. 6. Black hole mass distribution for Seyfert galaxies (top) and LLRGs (bottom).

values, i.e., $\log M_{\mathrm{BH}} \sim 7.5-9.5$. Compared to our Seyfert galaxy sample, there is an evident lack of objects with $\log M_{\mathrm{BH}}<7.5$. Note that most of the $M_{\mathrm{BH}}$ estimates have been obtained by using the mass vs. stellar velocity dispersion relation. Woo \& Urry (2002) have compared $M_{\mathrm{BH}}$ estimates derived from direct and indirect measurements of the stellar velocity dispersions for a sample of radio galaxies. The two methods agree on average to within $10 \%$, however the rms scatter is a factor of 4 . In this work, radio galaxies are mainly hosted by massive black hole hosts, even though the Seyfert and LLRGs' distributions overlap. This is in agreement with the results found by Sikora et al. (2006), Laor (2003), and Wu \& Han (2001). However, the role played by selection effects on this is still unclear. Indeed large compilations of data have shown that $M_{\mathrm{BH}}$ for radio-quiet and radio-loud objects show similar distributions, with several radioloud objects having $M_{\mathrm{BH}} \leq 10^{8} M_{\odot}$ (Woo \& Urry 2002; Ho 2002; Wang et al. 2006).
The relation between radio loudness and host properties has also been pointed out by Capetti \& Balmaverde (2006). They have found that the radio-loudness parameter in AGN hosted by early-type galaxies depends on the host's brightness profile, where core galaxies only host radio-loud AGN and radio-quiet AGN are found in power-law galaxies. Since the brightness profile is determined by the galaxy's evolution and merger history, the radio-loudness is therefore related to those basic parameters such as mass and spin, which are probably triggered by mergers.

An important role is also played by the accretion rate (Laor 2003). Originally, Ho (2002) found a trend of increasing radio loudness with decreasing Eddington ratio. In the left panel of Fig. 7 we show the radio loudness versus the Eddington ratio expressed as $L_{2-10 \mathrm{keV}} / L_{\text {Edd }}$. The Seyfert and LLRGs seem to confirm this trend, which could suggest that the formation of a jet in LLAGN is related to the accretion rate similarly to what is observed in XRBs. However, Sikora et al. (2006), by using samples of both high and low luminosity AGN, found two separate trends followed by radio-quiet and radio-loud AGN, and proposed a model that includes the black hole spin as one of the fundamental parameters, together with the mass, which determines the radio loudness in AGN, as is also previously conjectured by other authors (Blandford 1999; Meier 1999).

In the XRBs low/hard state the intermittency of the jet production is connected to the accretion rate (Fender et al. 2004). Apparently, the similarity between the jet and flow energy production in XRBs and AGN is rejected by the slope of the correlation we found for Seyfert galaxies and LLRGs, which differs from those found by Gallo et al. (2003) for low/hard state XRBs, $L_{R} \propto L_{\mathrm{X}}^{0.7}$. However, several authors were motivated to unify the central engine mechanisms in galactic black holes (GBHs) and super massive accreting black holes (SMBHs) (Maccarone et al. 2003; Merloni et al. 2003; Falcke et al. 2004). The observation of a correlation between the X-ray, radio, and black hole mass valid both for GBH and SMBH (the so-called "fundamental plane of black hole activity") has different interpretations. Merloni et al. (2003) suggest that the X-ray emission is produced by a radiatively inefficient accretion flow (ADAF), while the radio emission originates in a relativistic jet. Given the observed 

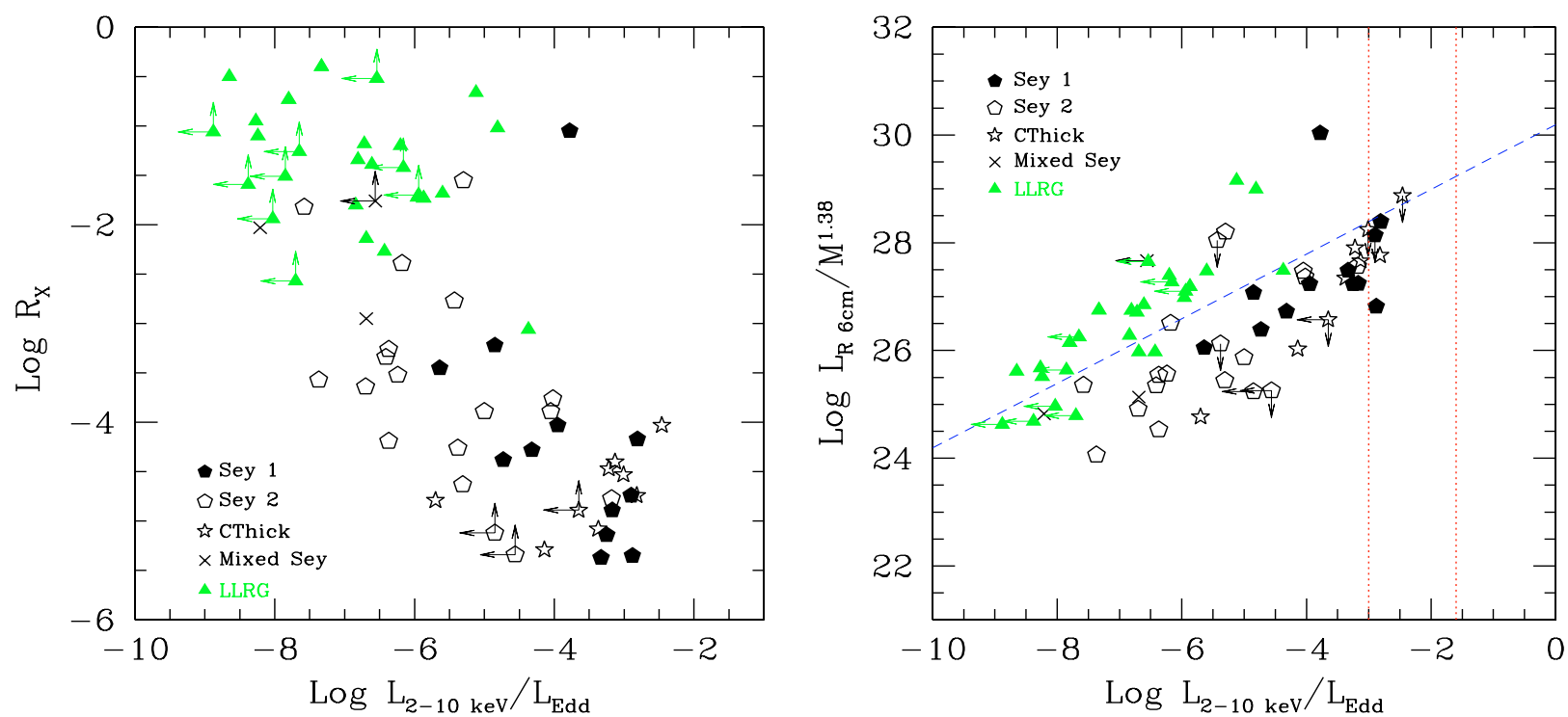

Fig. 7. Left panel: X-ray radio loudness versus Eddington ratio $L_{2-10 \mathrm{keV}} / L_{\mathrm{Edd}}$. Right panel: radio luminosity and $M_{\mathrm{BH}}^{1.38}$ ratio (from the fundamental plane equation in Merloni et al. 2003) versus the Eddington ratio.

correlations, the flow and the jet are strongly coupled. The fundamental plane equation also implies that the radio loudness should increase as the accretion rate decreases, and that the radio-loud and radio-quiet dichotomy should appear only at high accretion rates, similarly to what is observed in the high/very high transition in GBH.

In Fig. 7 (right panel), we plot the ratio between the radio luminosity and $M_{\mathrm{BH}}^{1.38}$ (the latter scaling is derived from the fundamental plane equation in Merloni et al. 2003) versus the Eddington ratio. The objects considered in the Merloni et al. (2003) sample collapse into a single track in this space (see Fig. 7 in Merloni et al. 2003). In our case, LLRG and the Seyfert galaxy follow two separate tracks indicating that a dichotomy is still observed down to low Eddington ratios. The region between the two vertical lines corresponds to the $L_{2-10 \mathrm{keV}} / L_{\mathrm{Edd}}$ values where the switch in the accretion mode is expected and where the radiatively efficient regime is dominant. According to this model, most of the Seyfert galaxies and all LLRGs should accrete in an inefficient radiative regime. Despite the fact that few Seyferts populate the "efficient accretion" zone, we do not observe any hint of a switch in the accretion mode above $L_{2-10 \mathrm{keV}} / L_{\mathrm{Edd}}>10^{-3}$. Moreover most Seyfert galaxies of this sample, in particular those of type 1 , have spectral characteristics inconsistent with models of low radiative efficiency, such as an X-ray power-law slope steeper than those predicted by ADAF models and/or the presence of an FeK line that accretion thin flows are unable to produce.

We overlaid the fundamental plane equation by Merloni et al. (2003) (short-dashed line in Fig. 7, right panel) derived as a function of $\log L_{2-10 \mathrm{keV}} / L_{\mathrm{Edd}}$. It appears to be a good fit for the whole the Seyfert galaxy plus LLRG sample. However, Seyfert galaxies and LLRGs seem to show two different fundamental planes. Indeed, Wang et al. (2006) have come to the same conclusion by investigating a sample of broad line SDSS AGN, both radioquiet and radio-loud. They have compared fluxes at $1.4 \mathrm{GHz}$ and $0.1-2.4 \mathrm{keV}$ with $M_{\mathrm{BH}}$ measurements. On the basis of their results these authors have suggested that radio-loud sources may obey the same correlation as radio-quiet sources, only shifted by a different factor depending on the radio-loudness parameter.
They also suggest that the beaming effects significantly alter the radio luminosity in radio-loud AGN, concluding that the fundamental plane in these sources is apparently unreliable if this effect cannot be removed.

\section{Summary}

We have investigated the correlation bewteen X-ray and radio luminosities for a sample of Seyfert galaxies and low luminosity radio galaxies (LLRGs) having nuclear data both at X-ray and radio frequencies. The radio loudness parameter and its dependence on luminosity, black hole mass, and Eddington ratio have also been discussed. We summarize our results as follows:

- Nuclear 2-10 keV X-ray and core radio luminosities at $20 \mathrm{~cm}, 6 \mathrm{~cm}$, and $2 \mathrm{~cm}$ are significantly correlated in the sample of nearby Seyfert galaxies down to very low luminosities $\left(L_{2-10 \mathrm{keV}}>10^{38} \mathrm{erg} \mathrm{s}^{-1}\right)$, suggesting a strong coupling between the X-ray and the radio emission mechanisms. The former is commonly ascribed to a disk-corona system and the latter is likely associated with a core jet/outflow.

- The X-ray versus $6 \mathrm{~cm}$ radio luminosity correlations hold for nearly eight orders of magnitude in both Seyfert and LLRG samples. Interestingly, in the two samples we found a similar correlation slope $\left(L_{\mathrm{X}} \propto L_{R}^{0.97}\right)$ suggesting either common physical mechanisms in their nuclei or a combination of different mechanisms that end up producing a similar spectral slope. It has been suggested that the X-ray emission in LLRGs could have a common non-thermal origin as the radio emission, as for example due to synchrotron radiation from a relativistic jet (Balmaverde et al. 2006); however, it is unlikely that this is also valid for Seyfert galaxies, for which a contribution from the accretion-flow/hot-corona system is expected in X-rays.

- Both the classical radio loudness parameter and the X-ray one show a distribution in Seyfert galaxies which is different from that of LLRGs. According to the classical boundaries between radio-loud and radio-quiet AGN, most of the Palomar Seyfert galaxies and all LLRGs are on the radioloud side. However, if one redefines the boundaries in both 
parameters $\left(R_{\mathrm{X}}=-2.755 \pm 0.015\right.$ and $\left.R=-2.400 \pm 0.050\right)$, the difference in the radio power between the two classes of objects is recovered. The boundaries are then different with respect to those of luminous AGN and this could be ascribed to an intrinsic property of low luminosity AGN. We find, however, no dependence of the radio loudness parameter on the luminosity.

- Black hole masses in the Seyferts and LLRG samples are differently distributed. Early-type LLRGs host more massive black holes than Seyfert galaxies, which commonly reside in spiral galaxies. This result confirms previous findings (Laor 2000; Wu \& Han 2001; Sikora et al. 2006) that higher black hole masses are associated with higher radio loudness parameters. However, the radio-loudness is not only host galaxy morphology dependent, but other parameters should play a role such as the host's brightness profile (Capetti \& Balmaverde 2006), the accretion rate, and the black hole spin (Blandford 1999; Meier 1999; Sikora et al. 2006).

- We confirm an anti-correlation between the radio loudness and the Eddington ratio, i.e., AGN with lower $L_{2-10 \mathrm{keV}} / L_{\mathrm{Edd}}$ tend to be more radio-loud. There is, however, no clear evidence of a transition between a radiatively efficient to a radiatively inefficient accretion regime and the radio-loud/radioquiet dichotomy seems to hold to low luminosities and low Eddington ratios, implying that the accretion rate is not the unique parameter that triggers the radio loudness.

Acknowledgements. We thank Mauro Dadina for helpful suggestions. We are greatful to the referee for a careful reading of the manuscript and his suggestions. F.P. acknowledges support by a "Juan de la Cierva" fellowship. Financial support for F.P., X.B., and F.J.C. was provided by the Spanish Ministry of Education and Science, under project ESP2006-13608.

\section{References}

Abramowicz, M. A. 1997, LNP: Accretion Disks - New Aspects, 487, 320

Akritas, M. G., \& Siebert, J. 1996, MNRAS, 278, 919

Balmaverde, B., Capetti, A., \& Grandi, P. 2006, A\&A, 451, 35

Balmaverde, B., \& Capetti, A. 2006, A\&A, 447, 97

Bicknell, G. V. 2002, New Astron. Rev., 46, 365

Blandford, R. D. 1999, Astrophysical Discs - an EC Summer School, ASP Conf. Ser., 160, 265

Brinkmann, W., Laurent-Muehleisen, S. A., Voges, W., et al. 2000, A\&A, 356, 445

Canosa, C. M., Worrall, D. M., Hardcastle, M. J., \& Birkinshaw, M. 1999, MNRAS, 310,30

Caon, N., Capaccioli, M., \& D’Onofrio, M. 1994, A\&AS, 106, 199

Capetti, A., \& Balmaverde, B. 2006, A\&A, 453, 27

Cappi, M., Panessa, F., Bassani, L., et al. 2006, A\&A, 446, 459

Chiaberge, M., Capetti, A., \& Macchetto, F. D. 2005, ApJ, 625, 716

Chiaberge, M., Gilli, R., Macchetto, F. D., Sparks, W. B., \& Capetti, A. 2003, ApJ, 582, 645

Cirasuolo, M., Celotti, A., Magliocchetti, M., \& Danese, L. 2003, MNRAS, 346, 447

Dunlop, J. S., McLure, R. J., Kukula, M. J., et al. 2003, MNRAS, 340, 1095

Elvis, M., Soltan, A., \& Keel, W. C. 1984, ApJ, 283, 479

Elvis, M., Wilkes, B. J., McDowell, J. C., et al. 1994, ApJS, 95, 1

Elvis, M., Risaliti, G., Nicastro, F., et al. 2004, ApJ, 615, L25

Evans, D. A., Worrall, D. M., Hardcastle, M. J., Kraft, R. P., \& Birkinshaw, M. 2006, ApJ, 642, 96

Falcke, H., Körding, E., \& Markoff, S. 2004, A\&A, 414, 895

Fender, R. P., Belloni, T. M., \& Gallo, E. 2004, MNRAS, 355, 1105

Filho, M. E., Barthel, P. D., \& Ho, L. C. 2002, ApJS, 142, 223
Filippenko, A. V., \& Sargent, W. L. W. 1989, ApJ, 342, L11

Filippenko, A. V., Ho, L. C., \& Sargent, W. L. W. 1993, ApJ, 410, L75

Gallo, E., Fender, R. P., \& Pooley, G. G. 2003, MNRAS, 344, 60

Ghisellini, G., Haardt, F., \& Matt, G. 2004, A\&A, 413, 535

Giroletti, M., Taylor, G. B., \& Giovannini, G. 2005, ApJ, 622, 178

Gliozzi, M., Sambruna, R. M., Brandt, W. N., Mushotzky, R., \& Eracleous, M. 2004, A\&A, 413, 139

Guainazzi, M., Fabian, A. C., Iwasawa, K., Matt, G., \& Fiore, F. 2005, MNRAS, 356,295

Haardt, F., \& Maraschi, L. 1991, ApJ, 380, L51

Heckman, T. M., Ptak, A., Hornschemeier, A., \& Kauffmann, G. 2005, ApJ, 634, 161

Henri, G., \& Petrucci, P. O. 1997, A\&A, 326, 87

Hewett, P. C., Foltz, C. B., \& Chaffee, F. H. 2001, AJ, 122, 518

Ho, L. C. 1999, ApJ, 516, 672

Ho, L. C. 2002, ApJ, 564, 120

Ho, L. C. 2003, Active Galactic Nuclei: From Central Engine to Host Galaxy, ASP Conf. Ser., 290, 379

Ho, L. C., \& Peng, C. Y. 2001, ApJ, 555, 650

Ho, L. C., \& Ulvestad, J. S. 2001, ApJS, 133, 77

Ho, L. C., Filippenko, A. V., \& Sargent, W. L. 1995, ApJS, 98, 477

Ho, L. C., Filippenko, A. V., \& Sargent, W. L. W. 1997a, ApJS, 112, 315

Ho, L. C., Filippenko, A. V., \& Sargent, W. L. W. 1997b, ApJ, 487, 568

Ho, L. C., Feigelson, E. D., Townsley, L. K., et al. 2001, ApJ, 549, L51

Hummel, E., Fanti, C., Parma, P., \& Schilizzi, R. T. 1982, A\&A, 114, 400

Isobe, T., Feigelson, E. D., \& Nelson, P. I. 1986, ApJ, 306, 490

Ivezić, Ž., Menou, K., Knapp, G. R., et al. 2002, AJ, 124, 2364

Ivezić, Z., Richards, G., Hall, P., et al. 2004, AGN Physics with the Sloan Digital Sky Survey, ASP Conf. Ser., 311, 347

Kellermann, K. I., Sramek, R. A., Schmidt, M., Green, R. F., \& Shaffer, D. B. 1994, AJ, 108, 1163

Kellermann, K. I., Sramek, R., Schmidt, M., Shaffer, D. B., \& Green, R. 1989, AJ, 98, 1195

Komossa, S., Böhringer, H., \& Huchra, J. P. 1999, A\&A, 349, 88

Laor, A. 2003, ApJ, 590, 86

Maccarone, T. J., Gallo, E., \& Fender, R. 2003, MNRAS, 345, L19

Malzac, J., Jourdain, E., Petrucci, P. O., \& Henri, G. 1998, A\&A, 336, 807

Meier, D. L. 1999, ApJ, 522, 753

Merloni, A., Heinz, S., \& di Matteo, T. 2003, MNRAS, 345, 1057

Merritt, D., \& Ferrarese, L. 2001, MNRAS, 320, L30

Miller, L., Peacock, J. A., \& Mead, A. R. G. 1990, MNRAS, 244, 207

Nagar, N. M., Falcke, H., Wilson, A. S., \& Ulvestad, J. S. 2002, A\&A, 392, 53

Nagar, N. M., Falcke, H., \& Wilson, A. S. 2005, A\&A, 435, 521

Narayan, R., \& Yi, I. 1994, ApJ, 428, L13

Panessa, F., Bassani, L., Cappi, M., et al. 2006, A\&A, 455, 173

Pedlar, A., Unger, S. W., \& Dyson, J. E. 1985, MNRAS, 214, 463

Salvato, M., Greiner, J., \& Kuhlbrodt, B. 2004, ApJ, 600, L31

Sambruna, R. M., Gliozzi, M., Tavecchio, F., Maraschi, L., \& Foschini, L. 2006, ApJ, 652, 146

Schmitt, J. H. M. M. 1985, ApJ, 293, 178

Shastri, P., Wilkes, B. J., Elvis, M., \& McDowell, J. 1993, ApJ, 410, 29

Shakura, N. I., \& Sunyaev, R. A. 1973, A\&A, 24, 337

Sikora, M., Stawarz, L., Lasota, J.-P., et al. 2006, [arXiv: astro-ph/0604095]

Stetson, P. B., \& Gibson, B. K. 2001, MNRAS, 328, L1

Strittmatter, P. A., Hill, P., Pauliny-Toth, I. I. K., Steppe, H., \& Witzel, A. 1980, A\&A, 88, L12

Terashima, Y., \& Wilson, A. S. 2003, ApJ, 583, 145

Tremaine, S., Gebhardt, K., Bender, R., et al. 2002, ApJ, 574, 740

Visnovsky, K. L., Impey, C. D., Foltz, C. B., et al. 1992, ApJ, 391, 560

Wang, R., Wu, X.-B., \& Kong, M.-Z. 2006, ApJ, 645, 890

Wilson, A. S., \& Ulvestad, J. S. 1987, ApJ, 319, 105

White, R. L., Becker, R. H., Gregg, M. D., et al. 2000, ApJS, 126, 133

Woo, J.-H., \& Urry, C. M. 2002, ApJ, 579, 530

Worrall, D. M. 1997, Relativistic Jets in AGNs, 20

Worrall, D. M., Tananbaum, H., Giommi, P., \& Zamorani, G. 1987, ApJ, 313, 596

Wu, X.-B., \& Han, J. L. 2001, A\&A, 380, 31

Zamorani, G. 1986, Quasars, IAU Symp., 119, 223

Zezas, A., Birkinshaw, M., Worrall, D. M., Peters, A., \& Fabbiano, G. 2005, ApJ, 627,711 